Pacific Journal of Mathematics

METRIZABILITY IN NORMAL MOORE SPACES 


\title{
METRIZABILITY IN NORMAL MOORE SPACES
}

\author{
D. Reginald Traylor
}

\begin{abstract}
The purpose of this paper is to establish some metrizability properties of normal Moore spaces and normal, locally compact Moore spaces. Certain screenable subsets of complete normal Moore spaces are proved to be strongly screenable. Additional properties are related to screenability.
\end{abstract}

The question as to whether each normal Moore space is metrizable has received considerable attention. Bing [1] and Jones [4] have results which give much information about such spaces. In particular, Jones established [4, Theorem 5] that each normal, separable Moore space is metrizable, provided that $2^{\boldsymbol{N}_{0}}<2^{\boldsymbol{N}_{1}}$. His result is a corollary to another theorem, in the same paper, which Heath [3] has shown to be equivalent to the hypothesis that $2^{\aleph_{0}}<2^{\aleph_{1}}$. Recently, it has been proved [2, Theorem 2] that if there is a normal, separable, nonmetrizable Moore space then there is such a space which is also locally compact. There seems to be little more known about the metrizability of normal, locally compact Moore spaces than is known about normal Moore spaces.

This paper is devoted to establishing some metrizability properties of normal Moore spaces and normal, locally compact Moore spaces. Since each locally compact Moore space is complete, it perhaps should be noted that Younglove [8] has interesting results concerning metrizability of complete Moore spaces. Theorem 1 of this paper establishes a relation between screenability and Younglove's Property $Q$ [8] in complete Moore spaces. Theorems 3 and 4 establish that certain screenable subsets of complete, normal Moore spaces are strongly screenable. It is also proved that if $2^{\aleph_{0}}<2^{\aleph_{2}}$, then each normal, locally compact, nonmetrizable Moore space has the following property: A space has Property $P$ if for each open covering $H$ of $S$ there exists an uncountable discrete collection $G$ refining $H$ such that $G$ is not screenable, but each uncountable subcollection of $G$ contains an uncountable screenable subcollection.

The statement that $S$ is a Moore space means that there exists a sequence of collections of regions satisfying Axiom 0 and the first three parts of Axiom 1 of [5]. A Moore space is complete if there exists such a sequence satisfying all of Axiom 1. By a development is meant a sequence of collections of regions satisfying Axiom 0 and the first three parts of Axiom 1.

The statement that the collection $G$ of mutually exclusive point sets is (strongly) screenable means that there exists a sequence $H_{1}, H_{2}$, 
$H_{3}, \cdots$ of (discrete) collections of mutually exclusive domains such that no domain in any $H_{i}$ intersects two elements of $G$ and each element of $G$ is a subset of some domain of some $H_{i}$.

The statement that the discrete collection $G$ of point sets is collectionwise normal means there exists a collection $H$ of mutually exclusive domains in the space such that each element of $G$ is a subset of some element of $H$ but no element of $H$ intersects two elements of $G$. The discrete collection $G$ is collectionwise abnormal if no collection of domains in the space covering $G^{*}$ satisfies the notion of collectionwise normality.

Younglove's Property $Q$. A point set $M$ has Property $Q$ if for each open covering $G$ of the space $S$ there is a refinement $G^{\prime}$ of $G$ covering $S$ such that if $w_{1}, w_{2}, w_{3}, \cdots$ is a sequence of distinct elements of $G^{\prime},\left(A_{n}+B_{n}\right)$ is in $w_{n}$ and $A_{1}, A_{2}, A_{3}, \cdots$ converges to $P$, a point of $M$, then so must $B_{1}, B_{2}, B_{3}, \cdots$ converge to $P$.

THeOREM 1. If $M$ is an uncountable, discrete, screenable points set in a complete, normal Moore space, then $M$ has Property $Q$.

Proof. Suppose that $G$ is an open covering of $S$, no element of which contains two points of $M$. Then there exists a sequence $H_{1}$, $H_{2}, \cdots$ of collections of mutually exclusive domains which satisfies the notion of screenability with respect to $M$. For each $n$, let $M_{n}=M$. $\left[H_{1}^{*}-\left(H_{1}^{*}+\cdots+H_{n-1}^{*}\right)\right]$. There exist domains $D_{1}, D_{2}, \cdots$ such that $D_{i}$ contains $M_{i}$ but $D_{i}$ does not intersect $D_{j}$ unless $i$ is $j$.

For each $n$, denote by $H_{n}^{\prime}$ the collection to which $h^{\prime}$ belongs if and only if there exists an element $h$ of $H_{n}$ such that $h^{\prime}$ is $h \cdot D_{n}$. Then $H_{n}^{\prime}$ covers $M_{n}$ and no element of $H_{n}^{\prime}$ contains two points of $M$. Furthermore, if $P$ is a point of $M_{n}$ and $h$ of $H_{n}^{\prime}$ contains $P$, then $P$ is not a limit point of $\left(H_{n}^{\prime}-h\right)^{*}$, nor of $H_{m}^{\prime *}$ where $m$ is not $n$. It follows then, that if $w_{1}, w_{2}, \cdots$ is a sequence of distinct elements of $H_{i}^{\prime}$, and $A_{n}$ is in $w_{n}$, then $A_{1}, A_{2}, \cdots$ converges to no point of $M$. Let $D$ be a domain containing $S-\sum H_{i}^{\prime *}$ such that $\bar{D}$ does not intersect $M$. Denote by $G^{\prime}$ the collection to which the domain $g$ belongs if and only if for some positive integer $n, g$ is an element of $H_{n}^{\prime}$ or there is an element $h$ of $G$ such that $g$ is $h \cdot D$. Clearly, $G^{\prime}$ is an refinement of $G$ covering $S$ which satisfies Property $Q$ with respect to $M$.

Younglove proved [8, Theorem 3] that if $M$ is a subset of a complete Moore space which has Property $Q$, then exists a development satisfying Axiom $C$, stated below, at each point of $M$.

Axıom C. There exists a sequence $G_{1}, G_{2}, \cdots$ satisfying conditions 1,2 , and 4 of Axiom 1 of [5] along with the condition (3) if $A$ is a point of a region $R$ and $B$ is a point of $R$, there a positive exists integer $n$ 
such that if $x$ is a region of $G_{n}$ containing $A$, and $y$ is a region of $G_{n}$ intersecting $x$, then $x+y$ is a subset of $R$, and unless $B$ is $A, x+y$ does not contain $B$.

For the sake of completeness, and to further indicate the relation between Property $Q$ and metrizability in Moore spaces, a statement of Moore's metrization theorem is included [6]:

THEOREM 2 (Moore). A necessary and sufficient condition that a space $S$ satisfying Axiom 0 of [5] be metrizable is that it have a development satisfying Axiom $C$.

THEOREM 3. If $M$ is an uncountable, screenable, discrete subset of a complete, normal Moore space, then there exist a sequence $M_{1}$, $M_{2}, \cdots$ and a development $G_{1}, G_{2}, \cdots$ such that each $M_{i}$ is a subset of $M, \sum M_{i}$ is $M$ and if each of $p$ and $q$ is a point of $M_{i}$ and each of $g, h$, and $g^{\prime}$ is an element of $G_{i}$ such that $g$ contains $p, g^{\prime}$ contains $q$, then $h$ does not intersect both $g$ and $g^{\prime}$, unless $p$ is $q$.

Proof. It is shown in the proof of Theorem 1 that each discrete screenable set $M$ in a normal Moore space is collectionwise normal. Denote by $H$ a collection of mutually exclusive domains in the space satisfying that notion with respect to $M$. It follows from Theorem 1 and Younglove's result mentioned above that there is a development $G_{1}, G_{2}, G_{3}, \cdots$ which satisfies Axiom $C$ at each point of $M$. For each positive integer $n$, denote by $M_{n}$ the subset of $M$ to which $x$ belongs if and only if it is true that if $g$ and $g^{\prime}$ belong to $G_{n}, g$ contains $x$, and $g$ intersects $g^{\prime}$, then $g+g^{\prime}$ is a subset of the element of $H$ containing $x$. The sequence $M_{1}, M_{2}, M_{3}, \cdots$ satisfies the theorem.

THEOREM 4. If $M$ is an uncountable, discrete, screenable subset of a complete, normal Moore space, then there exists a sequence $M_{1}$, $M_{2}, \cdots$ such that each $M_{i}$ is strongly screenable and $\sum M_{i}$ is $M$.

Proof. Denote by $M_{1}, M_{2}, M_{3}, \cdots$ the sequence of point sets defined in the proof of Theorem 3. For each positive integer $n$ and each point $x$ of $M_{n}$, denote by $g_{x}$ some region of $G_{n}$ which contains $x$ and $H_{n}$ the collection to which $h$ belongs if and only if there is a point $y$ of $M_{n}$ such that $h$ is $g_{y}$. If each of $x$ and $y$ belongs to $M_{n}$, no region of $G_{n}$ intersects both $g_{x}$ and $g_{y}$. Thus each $M_{n}$ is strongly screenable.

THEOREM 5. If $S$ is a normal, nonmetrizable Moore space and $H$ is an open covering of $S$ then there is an uncountable discrete collection $G$ of mutually exclusive, closed point sets such that $G$ 
refines $H$ and $G$ is collectionwise abnormal.

Proof. A result due to Bing [1, Theorem 9] establishes that if $H$ is an open covering of $S$, there is a sequence $H_{1}, H_{2}, \cdots$ of discrete collections of closed point sets such that each $H_{i}$ refines $H$, no region of $G_{i}$ intersects two of the elements of $H_{i}$ and $\sum H_{i}^{*}$ is $S$.

It follows that some such $H_{i}$ for some such open covering $H$ must be collectionwise abnormal, else $S$ is screenable and, by another result due to Bing [1, Theorem 8], thus metrizable. Then some $H_{i}$ is uncountable and collectionwise abnormal.

THEOREM 6. If $S$ is a locally compact, normal, nonmetrizable Moore space then there is a locally compact, normal Moore space $S^{\prime}$ such that $S^{\prime}$ contains an uncountable subset $M$ which has no limit point and is collectionwise abnormal.

Proof. Assume that $H$ is an open covering of $S$, each element of which is compact. By Theorem 5, there is an uncountable, discrete collection $G$ of mutually exclusive, closed point sets such that $G$ refines $H$ and is collectionwise abnormal.

Denote by $S^{\prime}$ the space in which $P$ is a point if and only if $P$ is an element of $G$ or a point in $S-G^{*}$. Since each element of $G$ is compact, a result due to Worrell [7] establishes that $S^{\prime}$ is a Moore space. To prove that $S^{\prime}$ is normal, suppose that $N$ and $K$ are mutually exclusive, closed point sets. Then $N^{*}$ and $K^{*}$ are mutually exclusive closed point sets and there exists mutually exclusive domains $D_{N}^{*}$ and $D_{K}^{*}$ containing $N^{*}$ and $K^{*}$ respectively. Then $D_{N}$ and $D_{K}$ contain $N$ and $K$ respectively, in $S^{\prime}$.

Clearly, $G$ is collectionwise abnormal in $S^{\prime}$ and no domain containing it is metrizable.

THEOREM 7. If there is a locally compact, normal Moore space which is not metrizable then there is one with a development $G_{1}$, $G_{2}, \cdots$ which contains a domain whose boundary $B$ is a uncountable point set having no limit point such that $G_{1}, G_{2}, \cdots$ does not satisfy Axiom $C$ at any point of $B$.

Proof. By Theorem 6, there is a locally compact, normal Moore space containing an uncountable, discrete point set $M$ which is collectionwise abnormal. In fact, no domain containing $M$ is metrizable.

Suppose that $B$ is the subset of $M$ to which $x$ belongs if and only if there is a region $R$ containing $x$ such that the double star of $x$ with respect to each $G_{i}$ fails to lie in $R$. Then $M-B$ is collectionwise normal and $B$ satisfies the conditions of the theorem. 
THEOREM 8. If there is a locally compact, normal, nonmetrizable Moore space then there exists a normal Moore space $S$ with a development $G_{1}, G_{2}, \cdots$ such that $S=K+M$ where $K$ is a locally separable domain such that $G_{1}, G_{2} \cdots$ satisfies Axiom $C$ at each point of $K$ and $M$ is an uncountable, discrete point set such $G_{1}, G_{2}, \cdots$ satisfies Axiom $C$ at no point of $M$.

Proof. Since $S$ is locally compact, it is topologically complete. By a result due to Younglove [8, Theorem 1], there are development $G_{1}, G_{2}, \cdots$ and a dense subset $N$ of $S$ such that $G_{1}, G_{2}, \cdots$ satisfies $A$ xiom $C$ at each point of $N$. It follows from Theorem 7 that there is an uncountable, discrete subset $M$ of $S$ such that $G_{1}, G_{2}, \cdots$ does not satisfy Axiom $C$ at any point of $M$. Suppose that $K$ is $N \cdot(S-M)$. Then $K$ is locally separable since $S$ is locally compact. Indeed, $K+M$, with the relative topology, satisfies the theorem.

THEOREM 9. If there exists a normal, locally compact, nonmetrizable Moore space then there exists one which contains an uncountable, discrete point set $M$ such that either $M$ has Property $P$ or no uncountable subset of $M$ is screenable.

Proof. Under the hypothesis of the theorem, it follows from Theorem 6 that there exists a normal, locally compact, nonmetrizable Moore space $S$ which contains an uncountable, discrete collection of degenerate point sets which is collectionwise abnormal. If that collection is called $M$, then $M$ is clearly not screenable. Indeed, either $M$ contains an uncountable subcollection such that no uncountable subcollection of it is screenable, or $M$ has Property $P$. Then $S$ clearly satisfies the theorem.

THEOREM 10. If $S$ is a locally compact Moore space containing an uncountable subset $M$ which has no limit point and is such that no uncountable subset of $M$ is screenable, then there exists a locally compact, separable subspace $S^{\prime}$ of $S$ which contains $M$.

Proof. Suppose that $M$ is an uncountable, discrete subset of the locally compact Moore space $S$ such that no uncountable subset of $M$ is screenable. Denote by $w$ a well-ordering of the elememts of $M$ and by $H$ an open covering of $M$, each element of which is compact. Denote by $v$ a sequence, each element of which is a subset of an element of $H$ and which contains some point of $M$ such that the first term of $v$ contains the first term of $w$ and if $v^{\prime}$ is an initial segment of $v$ then the first term of $v$ following each term of $v^{\prime}$ is a compact 
region $R$ containing the first term of $w$ which is not a limit point of $\sum_{g \in v^{\prime}} g^{*}$ such that $R \cdot \overline{\sum_{g \in v} g^{*}}$ does not exist. If $v$ is uncountable, it follows that some uncountable subset of $M$ is screenable. So $v$ is only countable and each point of $M$ is a point of $\sum_{g \in v} g^{*}$ or limit point of $\sum_{g \in v} g^{*}$. But $\sum_{g \in v} g^{*}$ is separable since each region in the countable sequence $v$ is compact. It is obvious then, that $\sum_{g \in v} g^{*}+M$ is a separable subspace of $S$ which contains $M$. Then using an argument similar to that of Fitzpatrick and Traylor [2, Theorem 2], there is a locally compact, separable Moore space having the desired properties.

THEOREM 11. If $S$ is a normal, locally compact, nonmetrizable Moore space and $2^{\boldsymbol{N}_{0}}<2^{\boldsymbol{\aleph}_{1}}$ then $S$ has Property $P$.

Proof. If $S$ does not have Property $P$, if follows from Theorem 10 and Jones' result [4, Theorem 6] (that each subspace of a normal Moore space is normal) that there exists a normal, separable Moore space $S$ containing an uncountable subset which has no limit point. But Jones [4, Theorem 5] has proved that this is impossible, provided that $2^{\boldsymbol{N}_{0}}<2^{\boldsymbol{N}_{1}}$.

THEOREM 12. If there exists a normal, locally compact, nonmetrizable Moore space $S$ and $2^{\boldsymbol{N}_{0}}<2^{\boldsymbol{N}_{1}}$, then there exist a normal, locally compact, nonmetrizable Moore space $S^{\prime}$ and an uncountable sequence $w$ whose terms are points of $S^{\prime}$ such that (1) no countable subsequence of $w$ runs through $w,(2)$ if $M$ is the point set to which $x$ belongs if and only if $x$ is a term of $w$, then $M$ has no limit point and is not screenable, and (3) if $w^{\prime}$ is any initial segment of $w$ such that $w^{\prime}$ is not $w$ and $N$ is the subset of $S^{\prime}$ to which $x$ belongs if and only if $x$ is a term of $w^{\prime}$, then $N$ is screenable.

Proof. By Theorem 11, $S$ has Property $P$. Thus there exists a normal, locally compact, nonmetrizable Moore space $S^{\prime}$ which contains an uncountable subset $T$ such that $T$ has no limit point, is not screenable, but each uncountable subset of $T$ contains an uncountable, screenable subset. Denote by $v$ a well-ordering of $T$. Denote by $w$ the maximal initial segment of $v$ such that if $t$ is a term of $w$ and $N$ is the point set to which $x$ belongs if and only if $x$ is in $w$ and $t$ does not precede $x$, then $N$ is screenable. It is clear that $w$ is uncountable. If $w$ is not $v$ then the subset $M$ of $T$ to which $x$ belongs if and only if $x$ is in $w$ is not screenable. For assume that $M$ is screenable. Then if $t$ is the first term of $v$ following $w$, it follows that $M+t$ is screenable and $w$ is not maximal. Moreover, no countable subsequence of $w$ runs through $w$. For suppose that $u$ is a countable subsequence of $w$ such that if $t$ is a term of $w$, then $t$ 
precedes some term of $u$ in $w$. If $y$ is a term of $u$, denote by $M_{y}$ the subset of $T$ to which $x$ belongs if and only if $x$ is in $w$ and precedes $y$. Then $M_{y}$ is screenable by the definition of $w$. But $\sum_{y \in u} M_{y}$ is $M$ if $u$ runs through $w$ and, since the sum of countably many screenable sets is screenable, $M$ would be screenable. Thus no countable subsequence of $w$ runs through $w$ and the proof is complete.

\section{BIBLIOGRAPHY}

1. R. H. Bing, Metrization of topological space, Canad. J. Math. 3 (1951), 175-186.

2. Ben Fitzpatrick and D.R. Traylor, Two theorems on metrizability of Moore spaces (to appear in the Pacific J. Math.)

3. R. L. Heath, Separability and $\boldsymbol{\aleph}_{1}$-compactness, Notices of Amer. Math. Soc., Vol. 9, No. 1, Abstract 588-19.

4. F. B. Jones, Concerning normal and completely normal spaces, Bull. Amer. Math. Soc. 43 (1937), 671-677.

5. R. L. Moore, Foundations of point set theory Amer. Math. Soc. Colloquium Pub. No. 13, Providence, 1962.

6. - A set of axioms for plane analysis situs, Fund. Math. 25 (1935), 13-28.

7. J. M. Worrell, Concerning upper semi-continuous collections of mutually exclusive closed and compact point sets, Notices of Amer. Math. Soc., Vol. 9, No. 3, Abstract 590-44.

8. J. N., Younglove, Concerning dense metric subspaces of certain nonmetric spaces, Fund. Math. 48 (1959), 15-25.

Received August 24, 1963, and in revised form June 15, 1964. Theorems 11 and 12 were presented to the American Mathematical Society, August 27, 1963, under the title, On metrizability of locally compact, normal Moore spaces.

AUBURN UNIVERSITY

THE UNIVERSITY OF HOUSTON 



\section{PACIFIC JOURNAL OF MATHEMATICS}

\section{EDITORS}

\author{
H. SAMELSON \\ Stanford University \\ Stanford, California \\ J. P. JANS \\ University of Washington \\ Seattle, Washington 98105
}

\author{
J. DUGUNDJI \\ University of Southern California \\ Los Angeles, California 90007 \\ RICHARD ARENS \\ University of California \\ Los Angeles, California 90024
}

\section{ASSOCIATE EDITORS}
E. F. BECKENBACH
B. H. NeumanN
F. WOLF
K. YOSIDA

\section{SUPPORTING INSTITUTIONS}

\author{
UNIVERSITY OF BRITISH COLUMBIA \\ CALIFORNIA INSTITUTE OF TECHNOLOGY \\ UNIVERSITY OF CALIFORNIA \\ MONTANA STATE UNIVERSITY \\ UNIVERSITY OF NEVADA \\ NEW MEXICO STATE UNIVERSITY \\ OREGON STATE UNIVERSITY \\ UNIVERSITY OF OREGON \\ OSAKA UNIVERSITY \\ UNIVERSITY OF SOUTHERN CALIFORNIA
}

STANFORD UNIVERSITY

UNIVERSITY OF TOKYO

UNIVERSITY OF UTAH

WASHINGTON STATE UNIVERSITY

UNIVERSITY OF WASHINGTON

AMERICAN MATHEMATICAL SOCIETY CHEVRON RESEARCH CORPORATION TRW SYSTEMS

NAVAL ORDNANCE TEST STATION 


\section{Pacific Journal of Mathematics}

\section{Vol. 19, No. 1 \\ May, 1966}

A. R. Brodsky, The existence of wave operators for nonlinear equations... 1

Gulbank D. Chakerian, Sets of constant width................... 13

Robert Ray Colby, On indecomposable modules over rings with minimum condition....................................... 23

James Robert Dorroh, Contraction semi-groups in a function space ....... 35

Victor A. Dulock and Harold V. McIntosh, On the degeneracy of the Kepler

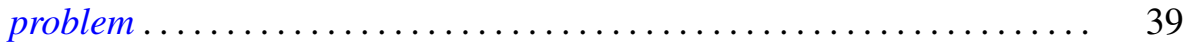

James Arthur Dyer, The inversion of a class of linear operators ......... 57

N. S. Gopalakrishnan and Ramaiyengar Sridharan, Homological dimension of Ore-extensions ................................. 67

Daniel E. Gorenstein, On a theorem of Philip Hall ................. 77

Stanley P. Gudder, Uniqueness and existence properties of bounded observables..................................... 81

Ronald Joseph Miech, An asymptotic property of the Euler function ....... 95

Peter Alexander Rejto, On the essential spectrum of the hydrogen energy and related operators ............................... 109

Duane Sather, Maximum and monotonicity properties of initial boundary

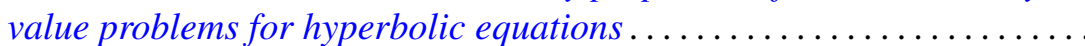

Peggy Strait, Sample function regularity for Gaussian processes with the parameter in a Hilbert space ........................... 159

Donald Reginald Traylor, Metrizability in normal Moore spaces ........... 175

Uppuluri V. Ramamohana Rao, On a stronger version of Wallis' formula ...............................

Adil Mohamed Yaqub, Some classes of ring-logics....... 